\title{
Detection and Termination of Broken-Spiral-Waves in Mathematical Models for Cardiac Tissue: A Deep-Learning Approach
}

\author{
Mahesh Kumar Mulimani ${ }^{1}$, Jaya Kumar Alageshan ${ }^{1}$, Rahul Pandit ${ }^{1}$ \\ ${ }^{1}$ Centre for Condensed Matter Theory, Department of Physics, Indian Institute of Science, Bangalore \\ 560012, India
}

\begin{abstract}
Defibrillation, the elimination of pathological waves of electrical activation in cardiac tissue, plays an important role in the elimination of life-threatening cardiac arrhythmias like ventricular tachycardia (VT) and ventricular fibrillation $(V F)$. We develop a deep-learning method, which uses a convolution neural network (CNN), to develop a new defibrillation scheme applicable in $2 D$ tisue. We begin by training our CNN with a huge dataset of spiral waves $(\mathcal{S})$ and non-spiral waves $(\mathcal{N S})$ that we obtain from our direct numerical simulations (DNSs) of a variety of mathematical models for the propagation of electrical waves of activation in cardiac tissue. Our trained CNN can distinguish between $\mathcal{S}$ and $\mathcal{N S}$ patterns; in particular, it also detects a broken spiral wave as $\mathcal{S}$. We demonstrate how to use our CNN to develop a heat map, from a brokenspiral-wave image, that yields the approximate locations of these spiral cores. We develop a defibrillation scheme that applies current, with two-dimensional (2D) Gaussian profiles of standard deviation $(\sigma)$, centred at square lattice sites $\left(N_{G} \times N_{G}\right)$ imposed on the simulation domain $(N \times N)$; the amplitudes of these Gaussians are taken from the heatmap. We explore the dependence of our Gaussian defibrillation scheme on a noisy image, which closely mimics the noisy optical image data.
\end{abstract}

\section{Introduction}

Pathological waves of electrical activation in cardiac tissue, such as spiral and broken-spiral-waves, are the mathematical analogs of ventricular tachycardia (VT) and ventricular fibrillation (VF), which are life-threatening cardiac arrhythmias [1]. Therefore, the termination of these waves is of paramount importance; this is referred to as defibrillation in a clinical setting. Traditional defibrillation methods employ electrical-voltage shocks to remove such wave behavior; although such shocks can remove arrhythmias, they can also damage cardiac tissue; this damage is potentially arrythmogenic. Thus, the development of low- amplitude defibrillation schemes has been a long-standing goal in this field. In-silico studies have played an important role in suggesting low-amplitude defibrillation schemes in both two-variable and realistic mathematical models for cardiac tissue (see Refs. [2-4] and references therein).

We approach this long-standing low-amplitude defibrillation problem from the perspective of deep learning. In particular, we develop a convolution neural netowrk $(\mathrm{CNN})$ that is capable of distinguishing between excitation-wave patterns with spiral waves $(\mathcal{S})$ and without spiral waves $(\mathcal{N S})$; to train this $\mathrm{CNN}$ we use our extensive dataset of images of excitation waves, which we generate via direct numerical simulations (DNSs) of a variety of partial-differential-equation (PDE) models for cardiac tissue (see, e.g., Eqns. (1) and (2) below). We then use our trained CNN to generate a heatmap for any broken-spiralwave image; this gives us information about the approximate positions of spiral cores. Fundamental to our defibrillation scheme is the observation that, a defibrillation current with 2D Gaussian profile $(\sigma=0.75 N)$ applied exactly at the spiral core eliminates a full-fledged spiral wave. We combine this observation and the heatmap to develop a defibrillation scheme which eliminates brokenspiral-wave. We explore the efficacy of our Gaussian defibrillation scheme and suggest experimental implementations of our scheme in cardiomyocyte-monolayer experiments.

\section{Materials and Methods}

We obtain the defibrillation-current profile in three steps: (i) We first train our CNN to distinguish between $\mathcal{S}$ and $\mathcal{N S}$; (ii) we then generate the heatmap $\mathcal{H}$ of the broken-spiral-wave pattern; and (iii) we then obtain the Hadamard product of $\mathcal{H}$ with the 2D Gaussian current profiles (see below) that we impose at every lattice point in our simulation domain.

To obtain the dataset for step (i), we employ mathematical models for cardiac tissue, which use nonlinear PDEs 
of the following reaction-diffusion type:

$$
\begin{array}{rlrl}
\frac{\partial V}{\partial t} & =D_{0} \nabla^{2} V+f(V, g) ; & \frac{\partial g}{\partial t}=\epsilon(V, g) h(V, g) ; \\
\frac{\partial V}{\partial t}=D_{0} \nabla^{2} V-\frac{I_{i o n}}{C_{m}} ; & I_{\text {ion }}=\sum_{i} I_{i} .
\end{array}
$$

We use the following two classes of models: (a) Twovariable models governed by eqn. 1, such as those in Refs. [5, 6]), where $V, g$, and $D_{0}$ are, respectively, the transmembrane potential (fast variable), the effective ionic gate (slow variable), and the diffusion constant; $f$ and $h$ are nonlinear functions of $V$ and $g$. (b) Realistic models with ion-channels governed by eqn. 2, such as those in Ref [7. 9], where $V, I_{i}$, and $C_{m}$ are the transmembrane potential, the ionic current for ion-channel $i$, and the membrane capacitance, respectively.

We use the forward-Euler method for time marching and a five-point stencil for the Laplacian for our spatial-finitedifference method. The temporal and spatial resolutions are $\Delta x$ and $\Delta t$, respectively (see respective models for the values), in our 2D square domain with $N \times N$ grid points ( $N=128,256,384,512,768$ and 1024 in our DNSs; typically we use $N=512$ ). We initiate the spiral wave by using the conventional S1-S2 cross-field protocol. Our DNSs yield the spatiotemporal evolution of $V$ that gives several patterns like spiral waves (Figure 1 a)), target waves (top right corner in Figure 1 (b)), plane waves (bottom left corner in Figure 1(b)), and states with spiral break-up (Figure 3).

We use the Deep Learning Toolbox in MATLAB R2018b to develop our CNN. We show a schematic diagram of this $\mathrm{CNN}$ in Figure 2. For details about the layers and units in the CNN see Refs. [10,11].

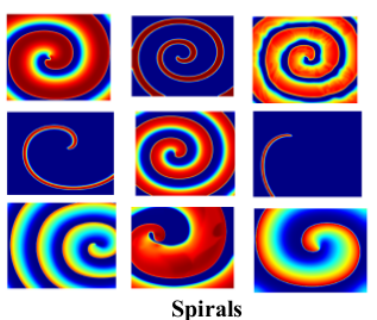

(A)

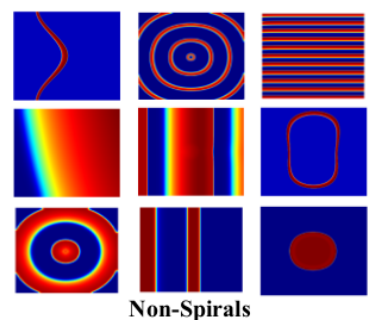

(B)
Figure 1. Illustrative pseudocolor plots of $V$ showing (a) single-spiral $(\mathcal{S})$ images and (b) images with no spirals $(\mathcal{N S})$

\section{Results}

\subsection{Training $\mathbf{C N N}$}

We use the normalised $\tilde{V}=\frac{\left(V-V_{\min }\right)}{\left(V_{\max }-V_{\min }\right)}, \tilde{V} \in[0,1]$, where the subscripts min and max denote the minimal and

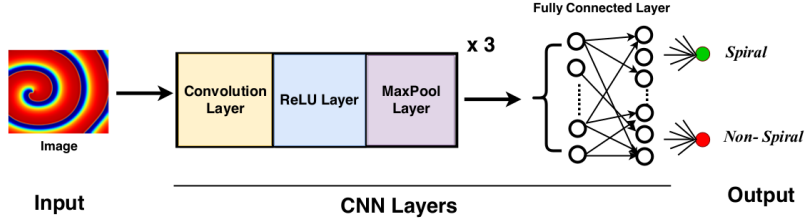

Figure 2. A schematic diagram of our CNN showing the Input, CNN layers, and Output (see Ref. [10] for details).

maximal values of $V$. Next we feed resized pseudocolor images of $\tilde{V}$ (we use a resized image with $32 \times 32$ points [11]) to train our CNN. If the output of our CNN predicts the class of the input image incorrectly, then we use a proxy cost function to rectify this error iteratively, until the CNN yields the correct output class; we ensure this for our CNN by minimizing the cross-entropy cost function (see Ref [10,11]). Our trained CNN achieves $99.7 \%$ accuracy in distinguishing between $\mathcal{S}$ and $\mathcal{N S}$. Even though we train our $\mathrm{CNN}$ with single-spiral-wave patterns, it manages to identify broken-spiral-wave patterns as $\mathcal{S}$. This is very useful for our defibrillation scheme.

\subsection{Heatmap $\mathcal{H}$}

We next generate the heatmap $\mathcal{H}(i, j)$ for a given pseudocolor plot of $V$, for those images detected as class $\mathcal{S}$ (especially broken-spiral-waves) as follows [11]:

$$
\mathcal{H}(i, j)=\frac{N_{p}}{N} \sum_{r=1}^{N / N p} \mathrm{CNN}\left(\chi_{i, j}^{r}\{V\}\right),
$$

$\forall i, j \in\{1,2, \ldots, N\}$; the arguments of $\chi_{i, j}^{r}$, the matrixresizing function, are the $32 r \times 32 r$ values of $V$ in a square of side $32 r$ centred at the point $(i, j)$, with $1 \leq i, j \leq N$ and $1 \leq r \leq\left(N /\left(4 \times N_{p}\right)\right)$; for the images here $N_{p}=32$. We use $\chi_{i, j}^{r}$ as an input into our CNN (Figure 2) and its output, 0 (for $\mathcal{N S}$ ) or 1 (for $\mathcal{S}$ ), is summed over $r$ to obtain $\mathcal{H}(i, j)$ for a given input pseudocolor plot of $V$; $\mathcal{H}(i, j) \in[0,1]$ is large if there is a spiral core near the point $(i, j)$. In Figure 3 we schematically depict how to generate a heatmap, from a pseudocolor plot of $V$, with a broken-spiral-wave pattern.

\subsection{Defibrillation current profile}

We can eliminate a single spiral wave by applying a current pulse with the following spatial profile: a symmetrical 2D Gaussian, with its centre at the core of the spiral wave and with equal widths in $x$ and $y$ directions, i.e., $\sigma_{x}=\sigma_{y}=\sigma$; such a Gaussian current pulse (see Ref. [11] has the virtue that it simultaneously depolarizes both the core of the spiral and the regions near the spiral arms and, eventually, eliminates the spiral. To eliminate a 


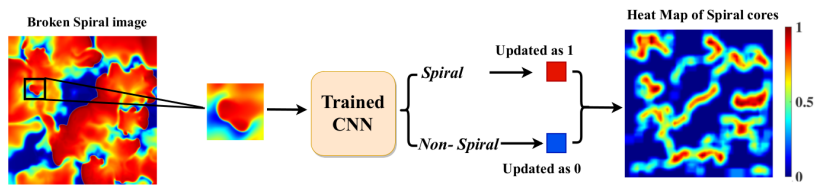

Figure 3. Schematic showing the method to obtain the heatmap $\mathcal{H}$. The pesudocolor image of broken-spiral-wave where, we scan from a lower pixel size of $32 r_{\min } \times 32 r_{\min }$ to maximum pixel size of $32 r_{\max } \times 32 r_{\max }$. A represntative pixel image is fed to CNN and the corresponding output is either 0 or 1 . This is repeated until $r=r_{\max }$, where after we obtain the heatmap $\mathcal{H}$. In this particular broken-spiral-wave data we use $r_{\min }=r_{\max }=2$.

broken-spiral-wave that has many spirals, we use a combination of Gaussian current pulses, to obtain a current profile that is large at spiral cores and small elsewhere. Specifically, we impose 2D Gaussians pulses centered at the sites of a 2D square lattice, with lattice parameter $a$; to ensure that these pulses have a large intensity at spiral cores, we use the Hadamard product of these Gaussian pulses with the heatmap $\mathcal{H}$ to obtain our final, defibrillation-current profile. For example, for the pattern with multiple-spiral wave in Figure 3), we (a) use a square lattice, of points $\left(i^{\prime}, j^{\prime}\right)$, with $0 \leq i^{\prime}, j^{\prime} \leq N_{G}$ and the lattice parameter $a=N_{G} \Delta x(\mathrm{~cm}),(\mathrm{b})$ impose a Gaussian current pulse $G\left(i^{\prime}, j^{\prime}\right)$ of width $\sigma$ and amplitude 1 on each lattice point, to obtain the following total, normalised contribution of these pulses, [at $(i, j)$, with $0 \leq i, j \leq(N-1)$, in the original image]:

$$
\begin{aligned}
\tilde{\mathcal{G}}(i, j) & =\sum_{i^{\prime}, j^{\prime}=0}^{N_{G}} G\left(i-i^{\prime} \frac{N}{N_{G}}, j-j^{\prime} \frac{N}{N_{G}}\right) ; \\
\mathcal{G}(i, j) & =\tilde{\mathcal{G}}(i, j) /\left[\tilde{\mathcal{G}}_{\text {max }}\right] ;
\end{aligned}
$$

here, $\tilde{\mathcal{G}}_{\text {max }}=\max _{(i, j)}[\tilde{\mathcal{G}}(i, j)]$. Our final current pulse, which we apply for $t_{d e f} \mathrm{~ms}$ at the point $(i, j)$, is given by the Hadamard product

$$
\mathcal{P}_{\mathcal{H}}(i, j)=I_{\text {def }} \mathcal{H}(i, j) \mathcal{G}(i, j),
$$

where $I_{d e f}$ is the maximum strength of the applied current, in our Gaussian defibrillation scheme, which we depict in Figure 4.

The efficacy our Gaussian scheme depends on the parameters such as $\sigma, a, I_{d e f}$ and $t_{d e f}$. We list these parameters (Table 11) for two illustrative Gaussian-control runs, GC1 and GC2. By comparing the results of such runs we find that, for large values of $a$, our Gaussian scheme is not successful in removing spiral waves; e.g.,the broken spiral waves are suppressed for the value of $a$ that we use in run $\mathrm{GC} 1$, but not for the value of $a$ in run GC2 (Table 1).
We refer the reader to Ref. [11] for a discussion of the dependence of our Gaussian defibrillation scheme on the parameters $a$ and $I_{d e f}$. At the qualitative level, we find that, for small (large) values of $a$, our Gaussian scheme is successful (not successful) in removing broken spiral waves. We list illustrative parameters in Table 11 for two cases [11].

\subsection{Training with the noisy image dataset}

In experiments, e.g., those that examine spiral waves in cardiomyocite layers [12], optical-image data are noisy. We have checked that our CNN-based Gaussian defibrillation scheme works even if we allow for such noise, whose strength can be measured by the signal-to-noise ratio (SNR). In particular, we have added noise to our spiral-wave images and shown them with thier respective heatmaps in Figure 5 for different values of SNR. We observe that, our CNN can faithfully generate the heatmap for SNR value greater than the threshlod value $\mathrm{SNR}_{\text {threshold }} \simeq 11.9$, for $1024 \times 1024$ simulation domain, and $\mathrm{SNR}_{\text {threshold }} \simeq 15.6$, for $256 \times 256$ simulation domain (not shown here). We observe that, $\mathrm{SNR}_{\text {threshold }}$ depends on the size of the simulation domain. We can obtain reliable heatmaps for SNR values much lesser than $\mathrm{SNR}_{\text {threshold }}$, by training our CNN with noisy image dataset.

\begin{tabular}{|l|l|c|c|c|c|}
\hline $\begin{array}{l}\text { Control } \\
\text { scheme }\end{array}$ & $N_{G}$ & $\begin{array}{c}a \\
(\mathrm{~cm})\end{array}$ & $\begin{array}{c}\sigma \\
(\mathrm{cm})\end{array}$ & $\begin{array}{c}I_{\text {def }} \\
(\mathrm{pA} / \mathrm{pF})\end{array}$ & $\begin{array}{c}t_{\text {def }} \\
(\mathrm{ms})\end{array}$ \\
\hline $\mathrm{GC} 1$ & 64 & 1.6 & 0.73 & 5 & 50 \\
$\mathrm{GC} 2$ & 96 & 2.4 & 0.73 & 5 & 50 \\
\hline
\end{tabular}

Table 1. Illustrative parameter values for our Gaussiancontrol scheme (GC1 and $\mathrm{GC} 2$ ).

\section{Conclusions}

We have shown how to use a deep-learning, CNNbased method to detect patterns with $(\mathcal{S})$ and without spiral waves $(\mathcal{N S})$, which we obtain from extensive DNSs of several mathematical models for the propagation of waves of excitation in cardiac tissue. We have also demonstrated how our trained CNN can be used to develop a heatmap that has high intensity in regions with spiral cores. We have then used this heatmap to develop our low-amplitude Gaussian defibrillation scheme, which we have described above; this scheme eliminates broken-spiral-waves. Given that such waves are the mathematical analogs of lifethreateniing cardiac arrhythmias, like VT and VF, we hope our in silico studies will lead to experimental studies of our Gaussian scheme in, e.g., experiments on cardiomyocyte layers. Here, from an experimental state with multiple spiral waves, a heat map and a Gaussian defibrilla- 


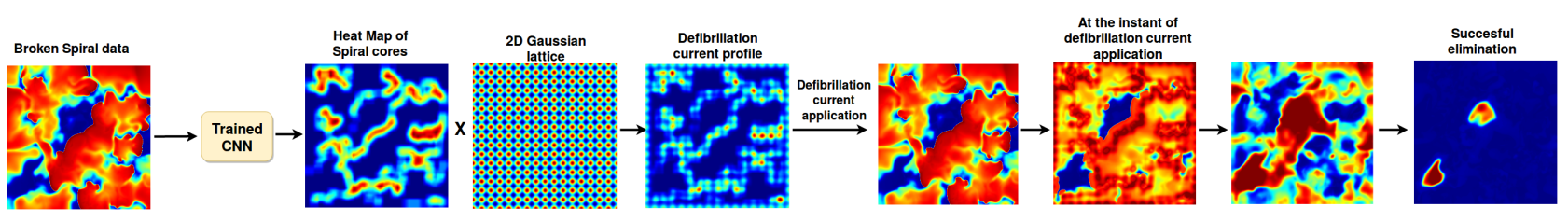

Figure 4. A schematic diagram showing how our 2D Gaussian defibrillation scheme (see text) eliminates a broken-spiralwave.

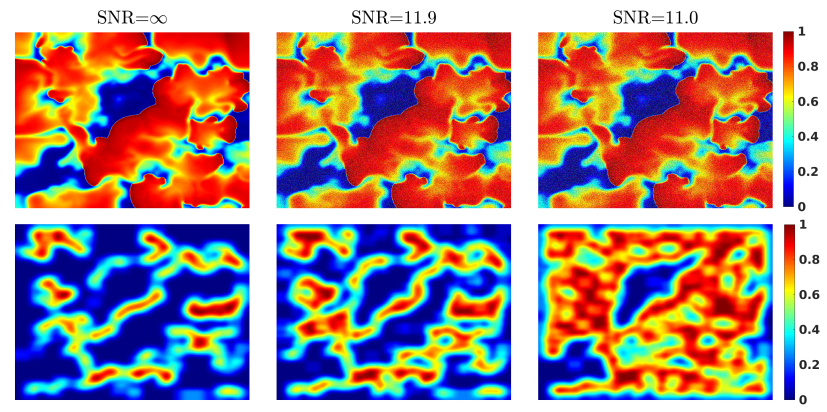

Figure 5. First row: Pseudocolor plots of $V$ with added noise and different values of the signal-to-noise ratio (SNR). Second row: Our CNN-generated heatmaps (see text) for the noisy-spiral images (directly above the heatmap).

tion scheme can be constructed by using the methods we have developed above. Our in-silico study has been limited to the detection and elimination of only 2D pathalogical waves. Also, our approach of defibrillation is through application of currents, which is difficult to realise, and is not the case with other defibrillation studies such as in Ref [13-15], where they apply electric fields for termination and is suitable for real-world application.

\section{Acknowledgements}

We thank DST (India) for the financial support and the Supercomputer Education and Research Centre (SERC, IISc Bangalore) for the computational resources.

\section{References}

[1] Mehra R. Global public health problem of sudden cardiac death. Journal of electrocardiology 2007;40(6):S118-S122.

[2] Osipov GV, Collins JJ. Using weak impulses to suppress traveling waves in excitable media. Physical Review E 1999;60(1):54.

[3] Rappel WJ, Fenton F, Karma A. Spatiotemporal control of wave instabilities in cardiac tissue. Physical review letters 1999;83(2):456.

[4] Sinha S, Pande A, Pandit R. Defibrillation via the elimination of spiral turbulence in a model for ventricular fibrillation. Physical review letters 2001;86(16):3678.
[5] Barkley D. A model for fast computer simulation of waves in excitable media. Physica D Nonlinear Phenomena 1991; 49(1-2):61-70.

[6] Aliev RR, Panfilov AV. A simple two-variable model of cardiac excitation. Chaos Solitons Fractals 1996;7(3):293301.

[7] Luo CH, Rudy Y. A dynamic model of the cardiac ventricular action potential. ii. afterdepolarizations, triggered activity, and potentiation. Circulation research 1994; 74(6):1097-1113.

[8] Ten Tusscher KH, Panfilov AV. Alternans and spiral breakup in a human ventricular tissue model. American Journal of Physiology Heart and Circulatory Physiology 2006;291(3):H1088-H1100.

[9] O'Hara T, Virág L, Varró A, Rudy Y. Simulation of the undiseased human cardiac ventricular action potential: model formulation and experimental validation. PLoS computational biology 2011;7(5):e1002061.

[10] Nielsen MA. Neural networks and deep learning, volume 25. Determination press USA, 2015.

[11] Mulimani MK, Alageshan JK, Pandit R. Deep-learningassisted detection and termination of spiral- and brokenspiral waves in mathematical models for cardiac tissue. 2019 ;

[12] Umapathy K, Nair K, Masse S, Krishnan S, Rogers J, Nash MP, Nanthakumar K. Phase mapping of cardiac fibrillation. Circulation Arrhythmia and Electrophysiology 2010; 3(1):105-114.

[13] Luther S, Fenton FH, Kornreich BG, Squires A, Bittihn P, Hornung D, Zabel M, Flanders J, Gladuli A, Campoy L, et al. Low-energy control of electrical turbulence in the heart. Nature 2011;475(7355):235.

[14] Otani NF. Termination of reentrant cardiac action potential propagation using far-field electrical pacing. IEEE Transactions on Biomedical Engineering 2011;58(7):2013-2022.

[15] Hornung D, Biktashev V, Otani N, Shajahan T, Baig T, Berg S, Han S, Krinsky V, Luther S. Mechanisms of vortices termination in the cardiac muscle. Royal Society open science 2017;4(3):170024.

Address for correspondence:

Mahesh Kumar Mulimani

Department of Physics, Indian Institute of Science,

Bangalore-560012, Karnataka, India.

maheshk@iisc.ac.in 